\title{
ARTICLE
}

Received 12 Jun 2013 | Accepted 4 Mar 2014 | Published 26 Mar $2014 \quad$ DOl: 10.1038/ncomms4536

\section{Controllable fatigue cracking mechanisms of copper bicrystals with a coherent twin boundary}

\author{
L.L. Li', Z.J. Zhang ${ }^{1}$, P. Zhang ${ }^{1}$, Z.G. Wang ${ }^{1} \&$ Z.F. Zhang ${ }^{1}$
}

High-angle grain boundaries are always the preferential fatigue cracking sites, while the intrinsic fatigue cracking mechanism of coherent twin boundary remains elusive. Here we systematically investigate the fatigue cracking behaviours of copper bicrystals with a coherent twin boundary as their sole internal boundary. It is found with direct experimental evidence for the first time that, unlike the random grain boundaries, the cracking behaviour of the twin boundary strongly depends on its orientation with respect to the loading direction. When the twin boundary is parallel or perpendicular to the loading direction, the fatigue cracks nucleate along the slip bands preferentially; when it is inclined at an angle to the loading direction, the fatigue crack is especially apt to nucleate along the twin boundary first. The controllable fatigue cracking mechanisms of the twin boundary may provide new and important implications for the optimized interfacial design of the high-performance materials.

\footnotetext{
${ }^{1}$ Shenyang National Laboratory for Materials Science, Institute of Metal Research, Chinese Academy of Sciences, 72 Wenhua Road, Shenyang 110016, China. Correspondence and requests for materials should be addressed to Z.F.Z. (email: zhfzhang@imr.ac.cn).
} 
$\mathrm{H}$ igh-angle grain boundaries (GBs) could enhance the strength of crystalline materials by hindering dislocation motion under monotonic loading ${ }^{1,2}$. However, the dislocation pileups could generate stress concentration and strain incompatibility at the GB, which facilitates the GB cracking under cyclic loading ${ }^{3-5}$. And it is found that the GB is always the preferential fatigue cracking site intrinsically rather than the slip band (SB) no matter whatever angle the $G B$ is inclined at in the bicrystals ${ }^{3}$. By suitable manipulation of the GB configuration, such as increasing the fractions of coincidence site lattice boundaries, the intergranular degradation could be improved and strong and ductile polycrystals would be developed $^{6,7}$. As a special coincidence site lattice boundary, the coherent twin boundaries (TBs) from the micro to angstrom scale are widely described both theoretically and experimentally to play a significant role in the plastic deformation of materials ${ }^{8-12}$. Due to the special crystallographic characteristics, the TB possesses specific interactions with the dislocations ${ }^{13}$. The TB could provide barrier to the dislocation motion like the conventional GB to enhance the strength ${ }^{9,10,13}$ and it is also a slip plane, which could accommodate ${ }^{10,11,13}$ or be penetrated by the dislocations $^{12,14,15}$ to improve the plasticity. On one hand, the incorporation of nanoscale TBs into materials can partly turn the trade-off relationship between the strength and ductility of the nano-grained materials ${ }^{9-12,16}$. On the other hand, the dislocations could glide in-between the twins, transfer across or slip parallel to the TB, which could be switched by changing the loading direction with respect to the $\mathrm{TB}$, resulting in different mechanical properties of the nano-twinned copper ${ }^{17-19}$. On the basis of the worldwide researches on the unidirectional deformation, it is urgent to investigate the fatigue cracking mechanisms of the $\mathrm{TB}$, which is of great significance in scientific research and engineering application. Different slip systems near the TB were observed in the fatigued coarse-grained copper ${ }^{20}$ and different TB-dislocation interactions could cause fatigue damage of different degrees to the TB in the polycrystals ${ }^{21,22}$. However, previous researches mainly employed polycrystalline aggregates with many kinds of planar defects. The existence of numerous grains with complicated stress conditions obscures the individual role of the TB in the mechanical properties. Therefore, this raises the intriguing scientific questions: what is the original role of the TB with cyclic loading and is there any controllability of the TB cracking distinct from the high-angle GB?

A series of grown bicrystals with a $\mathrm{TB}$ as its sole internal boundary were employed in the present investigation, wherein the angle between the TB plane and the loading direction of the twinned bicrystal $(\theta)$ is varied from $0^{\circ}$ to $90^{\circ}$ as depicted in Fig. 1a. The bicrystals with the TB parallel, perpendicular and inclined at $45^{\circ}$ to the loading direction, respectively, are representative cases and their primary slip systems are sketched in Fig. 1. The twin components are related with each other by reflection through the TB plane, which is set as plane ACD. The double Thompson tetrahedron is illustrated in Fig. If with the tetrahedrons above and below the TB representing slip systems in the matrix and twin grains, respectively ${ }^{13}$. When the TB is parallel to the loading direction, the two component grains have the same crystallographic orientation and same Schmid factor distribution. The slip systems with the highest Schmid factor in both the component grains are always the ones whose slip direction is parallel to the TB plane with the slip plane intersecting with the $\mathrm{TB}^{23}$ as depicted in Fig. 1c. The primary slip systems could be the slip vector AC on the slip plane $A B C$ in the matrix grain corresponding with the slip vector $\mathbf{A}^{\prime} \mathbf{C}^{\prime}$ on the slip plane $\mathrm{A}^{\prime} \mathrm{B}^{\prime} \mathrm{C}^{\prime}$ in the twin grain. This kind of slip system is denoted as $\mathrm{S} 1$ in this study. Usually, when the TB is inclined at $45^{\circ}$ to the loading direction, the primary slip planes in both component grains are parallel to the TB plane and their slip directions are parallel to each other as displayed in Fig. 1d. For instance, the slip vectors in both the component grains could be $A \mathbf{C}$ and $\mathbf{A}^{\prime} \mathbf{C}^{\prime}$ on planes $\mathrm{ACD}$ and $\mathrm{A}^{\prime} \mathrm{C}^{\prime} \mathrm{D}^{\prime}$, respectively. This kind of slip system is named as S2. As to the bicrystal with the TB perpendicular to the loading direction, naturally the loading directions of the two component grains are along the normal of the TB plane, $\langle 111\rangle$ direction, which is a multiple-slip orientation and six slip systems have the same Schmid factor of 0.272 . The primary slip system with slip plane and slip direction intersecting with the TB plane is named as S3. The representative example is sketched in Fig. 1e with BD on slip plane BCD and $\mathbf{B}^{\prime} \mathbf{D}^{\prime}$ on the slip plane $\mathrm{B}^{\prime} \mathrm{C}^{\prime} \mathrm{D}^{\prime}$ in both of the component grains. The above three kinds of twinned bicrystals manifest the three major interaction modes between incoming slip and TB. Generally, there might be arbitrary combinations of the above three slip systems. It is necessary to investigate the fatigue cracking behaviours of bicrystals whose primary slip systems differ from the above three kinds. Consequently five kinds of bicrystals with a TB inclined at $0^{\circ}, 30^{\circ}, 45^{\circ}, 60^{\circ}$ and $90^{\circ}$, respectively, were designed and applied in this study and the possible interactions between the TB and primary slip during the cyclic deformation are all taken into consideration using all the above twinned bicrystals.

Here we investigate the cyclic deformation and fatigue cracking behaviours of the twinned bicrystals with the help of scanning electron microscope (SEM) observation and electron channelling contrast (ECC) imaging method. The connections between the TB inclination angles and the fatigue cracking mechanisms are determined. When the TB is parallel or perpendicular to the loading direction, the SB cracks nucleate first; when the TB is inclined to the loading direction, the TB crack initiates preferentially. Obviously, this endows us with the control over the fatigue cracking behaviours of copper bicrystals with a TB.

\section{Results}

SEM and ECC results of coaxial twinned bicrystal. The slip morphologies with fatigue cracks and the corresponding dislocation arrangement of the twinned bicrystals are shown in Figs 2 and 3. The white arrows in the upper-right corner of the figures represent the loading direction of each specimen. Which kind of slip system was activated has been verified by combined consideration of the Schmid factors and the angles between the SBs and the loading direction on the two orthogonal specimen surfaces.

The deformation morphology and the dislocation arrangement of the fatigued bicrystal with the TB parallel to the loading direction are displayed in Fig. 2a,b, respectively. It can be seen from Fig. 2a that the SBs belonging to the S1 slip systems appear and no secondary SB is found ${ }^{23,24}$. The SBs can transfer across the TB with mirror symmetric relationship which is consistent with the schematic plot in Fig. 1c. The fatigue cracks nucleate preferentially along the primary SBs similar to single crystals ${ }^{3,5}$. Besides, some SB cracks exist in the grain interior and some SB cracks even pass through the TB as detected before ${ }^{23,24}$. As exhibited in Fig. 2b, some dislocation walls form in both of the component grains, which correspond with the surface persistent SBs like in the single crystal ${ }^{25}$ and some dislocation walls match up well across the $\mathrm{TB}^{14}$. On the whole, there is little difference in the SB distribution between areas near and far away from the TB and so is the dislocation arrangement. A single TB parallel to the loading direction has little impact on the cyclic deformation and fatigue cracking behaviours of the bicrystal and the TB would not form fatigue crack, which is different from the bicrystals or polycrystals with high-angle $\mathrm{GBs}^{3}$. 
a

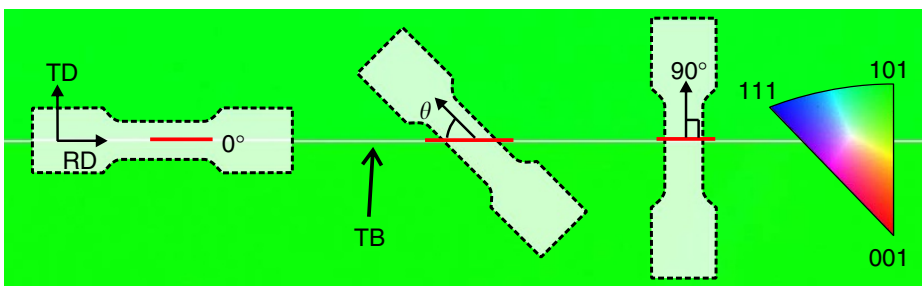

C

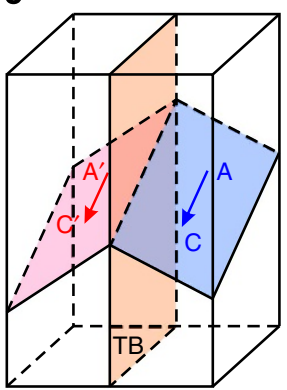

d

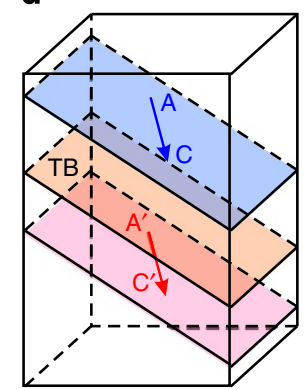

e

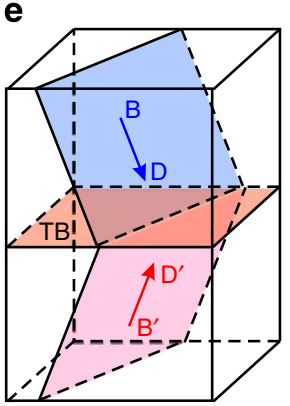

b

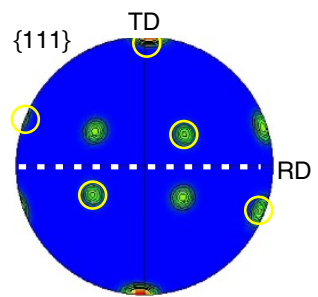

f

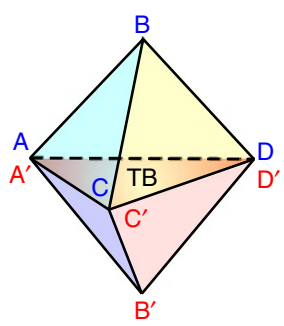

Figure 1 | The orientations and primary slip systems of the specimens. (a) Orientation and grain boundary (GB) map with the cutting ways of the specimens. (b) $\{111\}$ pole figure of the bicrystal; primary slip systems predicted by Schmid's law for the bicrystals with a twin boundary (TB) (c) parallel (d) inclined at $45^{\circ}$ and (e) perpendicular to the loading direction, respectively. (f) The double Thompson tetrahedron.
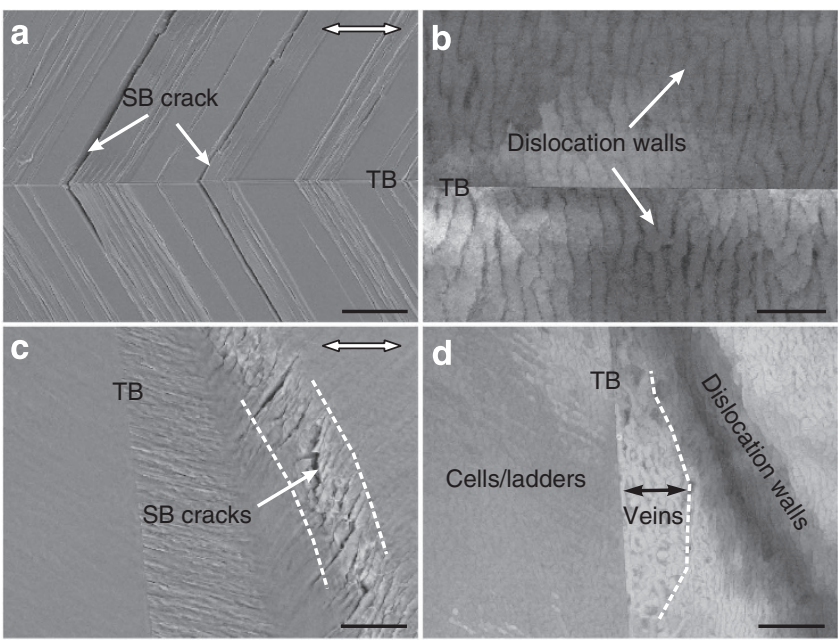

Figure 2 | Surface morphologies and dislocation arrangements of the bicrystals with a TB parallel or perpendicular to the loading direction.

(a) Slip morphologies with fatigue cracks of the bicrystal with the TB parallel to the loading direction. Scale bar, $50 \mu \mathrm{m}$. (b) Dislocation arrangements of the bicrystals with the TB parallel to the loading direction. Scale bar, $5 \mu \mathrm{m}$. (c) Slip morphologies with fatigue cracks of the bicrystal with the TB perpendicular to the loading direction. Scale bar, $10 \mu \mathrm{m}$.

(d) Dislocation arrangements of the bicrystals with the TB perpendicular to the loading direction. Scale bar, $10 \mu \mathrm{m}$.

SEM and ECC results of bicrystal with a perpendicular TB. When the TB is perpendicular to the loading direction, the S3 slip systems have the highest Schmid factors. The surface deformation morphology is shown in Fig. 2c. More than one slip systems are activated on the surface due to the multiple-slip orientation of the two component grains. The normal of the TB plane deviates from the loading direction slightly and then the slip morphology in the matrix grain is not exactly the same as that in the twin grain. The TB poses great barrier to the dislocation motion in this case $\mathrm{e}^{20}$ and with the cyclic deformation, the strain gradually concentrates in the grain interior. Thus it is observed that the fatigue cracks nucleate along the densely distributed SBs in the grain interior on one side of the TB as that found in single crystal ${ }^{26,27}$; in addition, there are already some fatal fatigue cracks formed along the SBs in areas close to the transition area of the specimen. As shown in Fig. 2d, on one side of the TB, there are some parallel dislocation walls corresponding with dense SBs in the grain interior while there are some veins adjacent to the TB corresponding with the sparse SBs. On the other side of the TB, dislocation cell or ladder structure exists, which is concerned with the coplanar slip systems as detected before ${ }^{28}$. It is clear that though there is pilling-up of dislocations at the TB which is perpendicular to the loading direction, the fatigue crack still initiates preferentially at the SBs rather than at the $\mathrm{TB}$, different from the high-angle $\mathrm{GBs}^{3}$.

SEM and ECC results of bicrystals with an inclined TB. Above all, when the TB is parallel or perpendicular to the loading direction, neither does the strain concentration nor does the fatigue cracking occur at the TB. The fatigue crack nucleates along the SBs in the grain interior. However, quite distinct fatigue cracking behaviours occur in the bicrystals with an inclined TB as displayed in Fig. 3. When the TB is inclined at $45^{\circ}$ to the loading direction, as demonstrated in Fig. 3a, SBs in both the component grains are parallel to the $\mathrm{TB}$, which is in accordance with the S2 slip systems sketched in Fig. 1d. Surprisingly, dense SBs are localized near the TB, generating a TB affected zone. The localized strain near the TB should be higher than that of the whole specimen ${ }^{29,30}$. The corresponding dislocation arrangement is shown in Fig. 3b. It is well-known that matrix veins form in the early stage of cyclic deformation and with cyclic deformation, the dislocation ladders appear ${ }^{31,32}$. There are already some dislocation ladders formed near the TB while it still exhibits as dislocation patches in the grain interior far away from the TB which is also an indication of strain localization. The dislocation ladders in the matrix grain have good correspondence across the TB with the ladders in the twin grain. With cyclic deformation, the dislocation configuration evolves more quickly near the TB which is consistent with the slip behaviour. Unlike the high-angle 

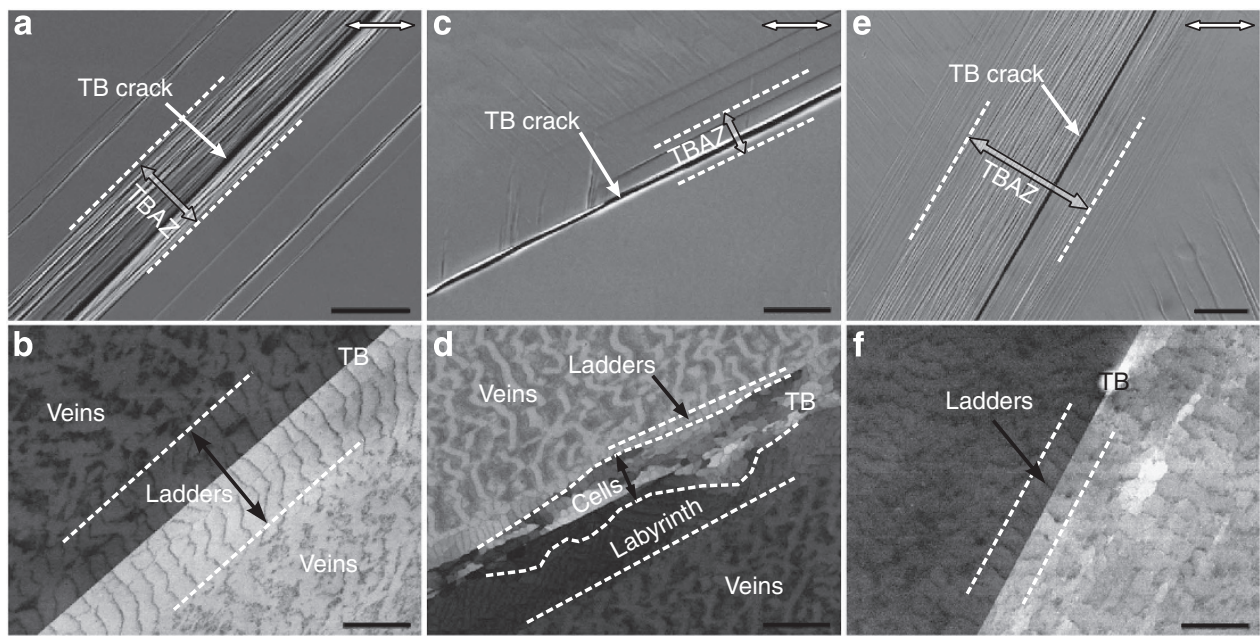

Figure 3 | Surface morphologies and dislocation arrangements of the bicrystals with an inclined TB. (a) Slip morphologies with fatigue cracks (scale bar, $20 \mu \mathrm{m}$ ) and (b) the dislocation arrangement of the bicrystal with a TB inclined at $45^{\circ}$ (scale bar, $5 \mu \mathrm{m}$ ). (c) Slip morphologies with fatigue cracks (scale bar, $10 \mu \mathrm{m}$ ) and (d) the dislocation arrangement of the bicrystal with a TB inclined at $30^{\circ}$ (scale bar, $5 \mu \mathrm{m}$ ). (e) Slip morphologies with fatigue cracks (scale bar, $20 \mu \mathrm{m}$ ) and (f) the dislocation arrangement of the bicrystal with a TB inclined at $60^{\circ}$ (scale bar, $5 \mu \mathrm{m}$ ).

$\mathrm{GB}$, there is no SB impingement on the TB, but the fatigue crack still nucleates first along the TB readily as indicated in Fig. 3a.

The slip morphologies with fatigue crack for the other two bicrystals with an inclined TB are displayed in Fig. 3c,e. The initial fatigue cracks form along the TB for both cases like the bicrystal with a TB inclined at $45^{\circ}$. Nevertheless, not all the SBs are localized at the TB, some SBs belonging to the S1 or S3 slip systems intersecting with the TBs also appear in the grain interior on one or two sides of the TB. When the TB is inclined at $30^{\circ}$ or $60^{\circ}$ to the loading direction, the Schmid factors of the S2 slip systems are not the highest in the two component grains. Unexpectedly, SBs parallel to the TB are activated in the TB affected zone during cyclic deformation as displayed in Fig. 3c,e which might be concerned with the strain compatibility and stress enhancement near the $\mathrm{TB}^{29,33}$. As shown in Fig. 3d with the ECC result of the bicrystal with a TB inclined at $30^{\circ}$, there are veins in the areas far away from the TB while there are some dislocation cells surrounding the $\mathrm{TB}$ demonstrating remarkable strain localization. The dislocation arrangement of the bicrystal with a TB inclined at $60^{\circ}$ is displayed in Fig. 3f. There are some dislocation ladders with good correspondence across the TB. It is also observed that there is a matchup between the dislocation ladders or cells across the TB in the bicrystal when the TB is inclined at $30^{\circ}$ to the loading direction and dislocation cells or labyrinths also form first near the TB which is inclined at $60^{\circ}$. Thus when the TB is inclined at $30^{\circ}, 45^{\circ}$ or $60^{\circ}$ to the loading direction, the S2 slip systems parallel to the TB plane are easily activated around the TB during cyclic deformation with pronounced fatigue cracking at TB eventually.

\section{Discussion}

Generally, the intergranular fatigue cracking is interpreted in the way of dislocation impingement with strain incompatibility and stress concentration ${ }^{3-5}$. Since the TB could be penetrated by the $\mathrm{S} 1 \mathrm{SBs}^{14,15}$, there is good strain compatibility near the TB which is parallel to the loading direction. So the TB shows higher fatigue cracking resistance than high-angle GB and the fatigue crack nucleates along the SB like in single crystals ${ }^{3,5}$. Whereas in the bicrystal with the TB perpendicular to the loading direction, the S3 SBs would pile up at the TB and could also penetrate through the TB partially ${ }^{34}$. The difference between the $\Omega$ of corresponding S3 slip systems in both the component grains is low, which generates high opportunity of dislocation penetration and good strain compatibility at the $\mathrm{TB}^{21,22}$. Then it shows the similar fatigue cracking behaviour to its component single crystal with preferential SB cracking and the TB perpendicular to the loading direction shows high fatigue cracking resistance.

For the bicrystals with an inclined TB, the S2 SBs are localized at the TB. Though they do not cause impingement to the TB, the S2 SBs play the crucial role in the TB shear fatigue cracking. Numerous vacancies could form along the TB or the SBs by the annihilation of edge dislocations with opposite signs during cyclic deformation ${ }^{5}$ different from unidirectional deformation. And the vacancies will concentrate and precipitate to form micro-cracks which would grow into the fatigue cracks afterward ${ }^{35}$ as illustrated in Fig. 4. The increase of the vacancy density $\mathrm{d} \rho_{\mathrm{v}}$ in an increment of shear strain $\mathrm{d} \gamma$ could be estimated as the density of annihilated edge dislocations $\mathrm{d} \rho_{\mathrm{e}}^{-5}$ :

$$
\mathrm{d} \rho_{\mathrm{v}}=\mathrm{d} \rho_{e}^{-}=2 \rho_{\mathrm{e}} y_{\mathrm{e}} \frac{\mathrm{d} \gamma}{\mathbf{b}}
$$

wherein, $\rho_{\mathrm{e}}$ is the dislocation density in the persistent SB walls and $y_{\mathrm{e}}$ is the distance for two edge dislocation to annihilate and $\mathbf{b}$ denotes the Burgers vector. According to the Taylor's equation ${ }^{36}$, it could be derived that:

$$
\rho_{\mathrm{e}}=\tau^{2} /(\alpha G \mathbf{b})^{2}
$$

wherein $\alpha$ is constant, $G$ is the shear modulus, $\tau$ is the cyclic shear stress. Then Equation (1) could be transformed into:

$$
\mathrm{d} \rho_{\mathrm{v}}=2 y_{\mathrm{e}} \tau^{2} \mathrm{~d} \gamma /\left(\alpha^{2} G^{2} \mathbf{b}^{3}\right) .
$$

Usually the strain is not homogeneously distributed along the whole specimen and there is strain localization in the fatigued materials. It is believed that the dislocations in the SBs spread homogeneously all over the specimen and so do the vacancies as illustrated in Fig. 4a,b. However, it could be derived from the present experimental results that the dislocations tend to transfer to the TB plane by cross-slip as illustrated in Fig. $4 \mathrm{c}$ and the TB allows room for the dislocation storage to accommodate high plastic strain ${ }^{37}$. Numerous vacancies would generate on the TB or in the close proximity of the TB ascribed to the annihilation of the dislocations of the S2 slip systems. Subsequently the vacancy reaches a much higher density locally on the TB than the SBs in the grain interior as shown in Fig. $4 \mathrm{~d}$. Therefore, the degree of the 

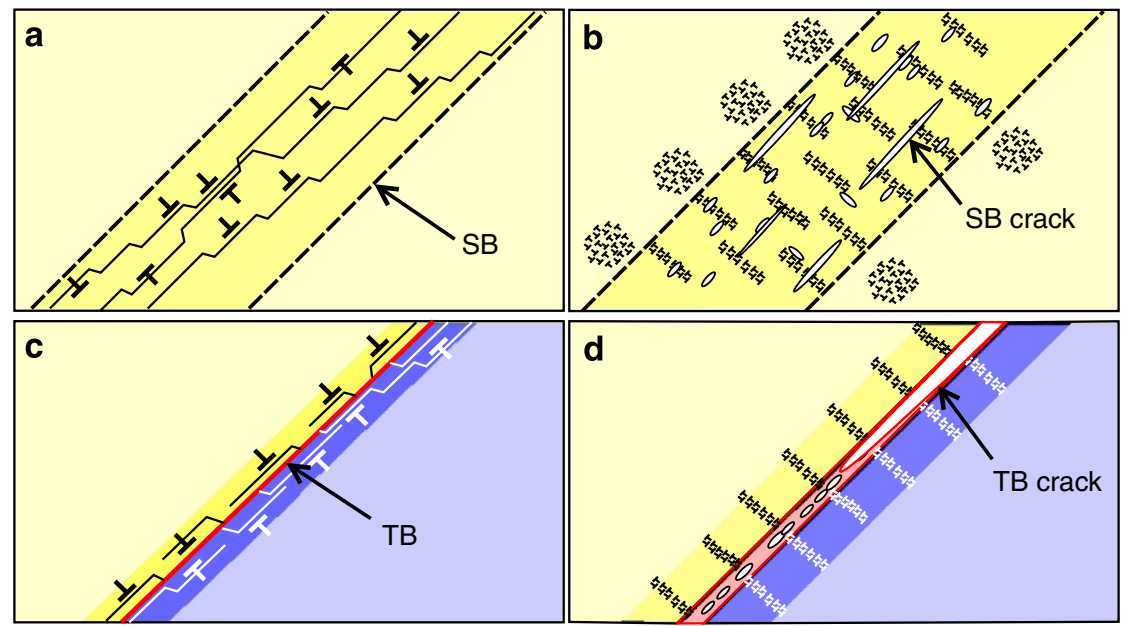

Figure 4 | The sketch for the dislocation motion and the crack formation along the slip band and the TB. (a) Dislocation cross-slip in the slip bands (SBs) in the grain interior. (b) The vacancies aggregate in the SBs to form the SB crack. (c) The dislocations from the two component grains transfer to the TB by cross-slip. (d) The vacancies aggregate on the TB to form the TB crack.

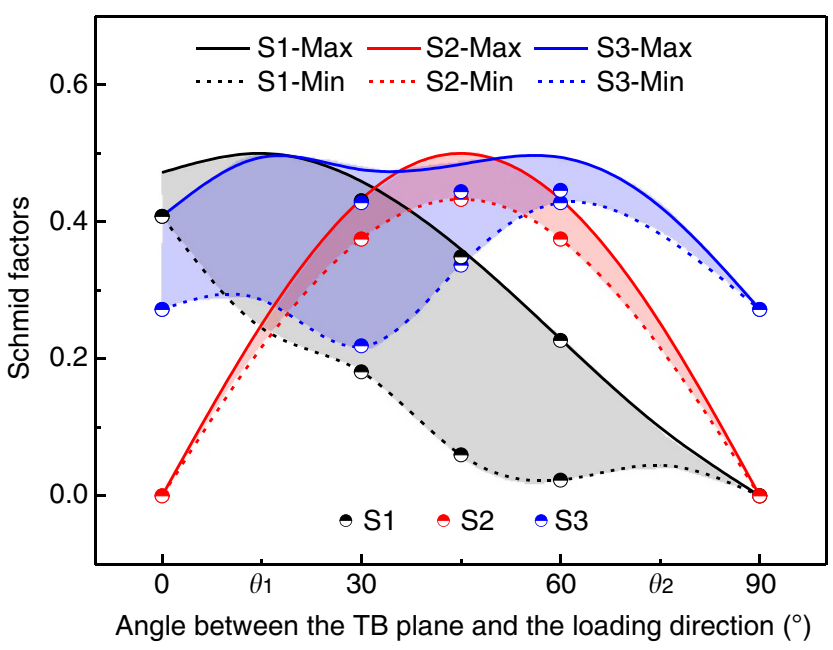

Figure 5 | The Schmid factor distribution of the twinned bicrystals.

The solid and dashed lines with different colors represent the value ranges for the Schmid factors of the three kinds of slip systems, respectively, in both of the component grains with the TB inclinations changing from $0^{\circ}$ to $90^{\circ}$. The Schmid factors of the three kinds of slip systems for the employed bicrystals are illustrated by half-filled circles with corresponding colors.

vacancy aggregation along the TB could be expressed as:

$$
\mathrm{d} \rho_{\mathrm{v}}=2 y_{\mathrm{e}} \eta \tau^{2} \mathrm{~d} \gamma /\left(\alpha^{2} G^{2} \mathbf{b}^{3}\right) .
$$

The parameter $\eta$ is introduced to characterize the degree of the strain localization. The increase in the vacancy density per cycle $\Delta \rho_{\mathrm{v}}$ could be obtained by integration of Equation (4). When the vacancy density along the interface increases to the critical value, $C$, the fatigue crack initiates. The critical vacancy density could be considered as the same for the SB and TB cracks in one bicrystal specimen because both of them nucleate along the $\{111\}$ plane. Thus, the critical cycles for the fatigue cracking could be expressed as:

$$
N_{\mathrm{c}}=C / \int_{\sigma_{0}}^{\sigma} \mathrm{d} \rho_{\mathrm{v}} .
$$

The positions with high $\tau$ and $\eta$ are the preferential fatigue cracking sites with lower $N_{c}$. The actual cycles for the fatigue

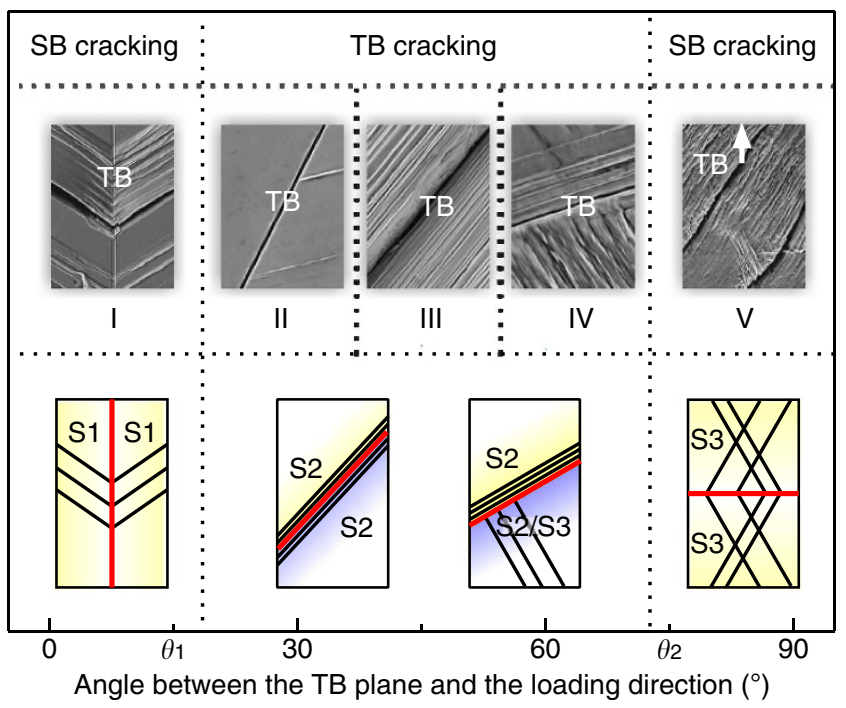

Figure 6 | General fatigue cracking mechanisms of the twinned bicrystals with all possible TB inclinations $\left(0^{\circ}\right.$ to $\left.90^{\circ}\right)$. The fatigue cracking behaviours are shown in the upper part of the figure and the schemes for the corresponding slip systems are displayed in the lower part. In regions I and V, S1 and S3 slip systems operate easily and there is good strain compatibility at the $T B$, therefore the fatigue cracks nucleate along the SBs first; in regions II-IV, the S2 slip systems operate easily and concentrate at the TB which leads to the preferential shear fatigue cracking at the TB.

cracking could not be given by the above analysis, but it could help explain which position would form fatigue cracks preferentially in a bicrystal with an inclined TB.

Apparently, S1 slip systems generate little damage to the TB parallel to the loading direction and so do the S3 slip systems to the TB perpendicular to the loading direction; while S2 slip systems bring shear damage to the TB which would lead to the final TB fatigue cracking. The TB orientation and the operated slip systems are interrelated and affect the fatigue cracking behaviours of the twinned bicrystals. The distribution ranges of the Schmid factors for the three kinds of slip systems are illustrated in Fig. 5 when the TB is inclined at different angles $\left(0^{\circ}-90^{\circ}\right)$ to the loading direction. The black solid and dashed 
lines represent the upper and lower limits of the Schmid factors for the S1 slip systems, respectively, while the red ones display that for the S2 slip systems and the blue ones show that for the S3 slip systems. Wherein, the Schmid factors of the three kinds of slip systems for the employed bicrystals are illustrated by halffilled circles with corresponding colours. Then by combining the calculation and experimental results, the fatigue cracking behaviours of the twinned bicrystals with all possible $\mathrm{TB}$ inclinations are summarized in Fig. 6.

In region I when $\theta$ is lower than $\theta_{1}$, due to the highest Schmid factors, the $S 1$ slip systems can be activated easily on both sides of the TB as the sketch shown in Fig. 6. While in region V when $\theta$ is higher than $\theta_{2}$, the S3 slip systems have the highest Schmid factor $\Omega$ and will operate in the grain interior. The S1 and S3 slip systems will undertake the main cyclic deformation in the two cases respectively and both of them could pass through the TB generating slight pilling-up of dislocations and good strain compatibility at the TB. During cyclic deformation, the vacancy generation prevails in the SBs which will lead to the final SB fatigue cracking preferentially as the SEM images shown in Fig. 6. It can be obtained that $\theta_{1}$ is lower than $25^{\circ}$ and $\theta_{2}$ is larger than $70^{\circ}$ on the basis of our available experimental results and the crystallographic analysis in Fig. 5, which need more quantitative studies in the future. In region III when $\theta$ is near $45^{\circ}$, S2 slip systems possess the highest Schmid factors and operate easily on both sides of the TB. In regions II and IV, the shear stress of S2 slip systems may not be the highest as in some of the present bicrystal specimens. Therefore S1 or S3 slip systems may also appear in the grain interior due to their relatively high Schmid factors. However, the S2 slip systems can always be easily activated in the vicinity of the TB with stress enhancement as evidenced by our experimental results. It could be inferred from Equation (4) that the local strain localization and higher stress would cause the higher vacancy concentration near the TB due to the annihilation of the dislocations from the S2 slip systems. Eventually, shear fatigue crack initiates along the TB preferentially in the three regions II-IV. Interestingly, the present TB cracking in the bicrystals originates from the shear damage caused by the S2 slips which obviously differs from the conventional GB cracking caused by slip impingement.

On the basis of the experimental results and analysis above, the dislocation behaviours and fatigue cracking mechanisms of the twinned bicrystal are found to vary with the TB inclination angles. The TBs in regions I and $\mathrm{V}$ exhibit higher fatigue cracking resistance under cyclic deformation and the SB cracks nucleate first. Besides, they can provide strengthening effect to the materials under unidirectional deformation since S1 and S3 slips become hard slip modes which can contribute to the higher yield stress of polycrystals ${ }^{17-19}$. In the central three regions II-IV, the shear TB crack nucleates preferentially and the S2 slip systems play the crucial role because they concentrate at the TB and bring shear damage to the TB. Meanwhile, the S2 slip systems become the soft slip mode and can lead to the lower strength of polycrystals with inclined TBs under unidirectional deformation ${ }^{11,12,17-19}$. Thus, the inclined TB could hardly strengthen materials and it is also the weakest site in the fatigued bicrystals. Therefore the fatigue performance of the materials could be improved through controlling the inclination of the TBs and it is important to prepare materials with highly textured TB. It has been documented that the TBs are normal to the growth direction during magnetron sputtering, electrodeposition or solution precipitation $^{8,17,38}$. There could also be TBs of pronounced meso-texture, which appeared after cold rolling and consequent thermal treatment ${ }^{39}$. These methods enable the control over the $\mathrm{TB}$ orientations in real samples. However, the specimen size, the twin thickness and the texture strength of TBs need to be improved by more studies in future.

In conclusion, the fatigue cracking behaviours of the copper bicrystals with a single TB was investigated. Quite distinct from the high-angle GB, the intrinsic role of $\mathrm{TB}$ varies with the $\mathrm{TB}$ inclination angle. The fatigue cracking behaviours of the bicrystals could be controlled by adjusting the TB orientation, which provides important implications for the interfacial engineering design of materials in the future. The current work unveils the specialty of the TB with cyclic loading which would also inspire scientific research about other special GB.

\section{Methods}

Sample preparation. Bulk copper bicrystal with a coherent TB as its sole internal boundary was grown from oxygen-free high-conductivity copper of $99.999 \%$ purity by Bridgman method. The crystallographic orientation was determined by electron backscatter diffraction. The inverse pole figure and GB map of the twinned bicrystal is displayed in Fig. 1a and the colour represents the orientation along the normal direction of the bicrystal with the colour key displayed as the inset. The same colour indicates that the normal direction of the two grains are the same and near $<110\rangle$ direction. The same normal direction of the component grains means that the TB is vertical along the thickness direction. As shown in Fig. 1b, the $\{111\}$ poles of the two grains are symmetrical about the TB plane (the white dashed line). The poles with a circle surrounding belong to the matrix grain while the rest poles represent the twin grain and the symmetric $\{111\}$ poles means that it is a coherent TB. The way in which the specimens were prepared from the bulk materials is illustrated in Fig. 1a. The size of the gauge section of the specimen is about $2.5 \times 2.5 \times 6 \mathrm{~mm}^{3}$. All the fatigue specimens were ground and electropolished carefully before cyclic deformation.

Experimental methods. The bicrystal specimens were cyclically deformed under loading control at room temperature in air and a triangle wave with a frequency of $1 \mathrm{~Hz}$ was applied with an Instron E1000 testing machine. More than three specimens for each kind of bicrystal were applied in the fatigue experiments. Tests in cyclic deformation were conducted under load control. The bicrystals were fatigued with zero mean stress at increased stress amplitude. The shear stress amplitude increased stepwise from $10 \mathrm{MPa}$ to about $32 \mathrm{MPa}$ and after that the shear stress amplitude was kept at $32 \mathrm{MPa}$ (corresponding to their cyclic saturation resolved shear stress ${ }^{40}$ ) with little adjustment. The total strain amplitudes reached at the final stress amplitudes of all the specimens are all calculated to be about $1 \%$ on the basis of the beam displacement detected by the testing machine. The fatigue specimens were cyclically deformed until fatigue cracks initiated either at the TBs or SBs which could be detected by the SEM. The cyclic numbers needed to detect the SB cracks are higher than $5 \times 10^{4}$ in the bicrystal with a parallel $\mathrm{TB}$, the longest in all the fatigue specimens. For the bicrystals with the TB inclined to the loading direction, the cyclic numbers are the lowest, around $2 \times 10^{4}$. It is about $3 \times 10^{4}$ cycles at which the fatigue crack was observed in the bicrystal with a TB perpendicular to the loading direction. The resolved shear stress of the bicrystal with a perpendicular TB increased monotonously with increasing cyclic number without saturation phenomenon as that in a cyclically deformed $<111>$ copper single crystal ${ }^{27,30}$. Thus the nominal resolved shear stress amplitude has been raised to about $48 \mathrm{MPa}$ until the fatigue cracks were detected.

Slip morphology and dislocation arrangement observation. The slip morphologies and fatigue cracks of the specimens were observed by Zeiss Supra- 35 SEM. After SEM observation on the surface, the specimens were ground and electropolished again and examined by ECC imaging technique ${ }^{3,41}$ to detect the dislocation arrangement near the TB. A channelling contrast is produced by using the conventional image scanning with a back-scattered electron detector. There are four quadrant-back-scattering-detectors and there is no tilt of the specimens or the incident beam. The four quadrant-back-scattering-detectors were all set as negative in this ECC observation so the ECC images are similar in appearance to the TEM bright field micrographs. The incident electron beam is along $<110\rangle$, the normal direction of the specimen. Unlike the ECC observation on materials with low dislocation density ${ }^{42}$, the diffraction conditions could be relaxed in the present study in detecting the fatigued dislocation arrangements ${ }^{43-46}$. The working distance is about $8 \mathrm{~mm}$ and the acceleration voltage is $20 \mathrm{kV}$ using an aperture of $60 \mu \mathrm{m}$.

\section{References}

1. Petch, N. J. The cleavage strength of polycrystals. J. Iron Steel Inst. 174, 25-28 (1953).

2. Hall, E. The deformation and ageing of mild steel: III discussion of results. Proc. Phys. Soc. B 64, 747-753 (1951).

3. Zhang, Z. F. \& Wang, Z. G. Grain boundary effects on cyclic deformation and fatigue damage. Prog. Mater. Sci. 53, 1025-1099 (2008). 
4. Laird, C. \& Smith, G. C. Initial stages of damage in high stress fatigue in some pure metals. Philos. Mag. 8, 1945-1963 (1963).

5. Essmann, U., Gösele, U. \& Mughrabi, H. A model of extrusions and intrusions in fatigued metals I. Point-defect production and the growth of extrusions. Philos. Mag. A 44, 405-426 (1981).

6. Watanabe, T. An approach to gain grain boundary design for strong and ductile polycrystals. Res. Mech. 11, 47-84 (1984).

7. Palumbo, G., King, P. J., Aust, K. T., Erb, U. \& Lichtenberger, P. C. Grain boundary design and control for intergranular stress-corrosion resistance. Scripta Metall. Mater. 25, 1775-1780 (1991).

8. Wang, J. W. et al. Near-ideal theoretical strength in gold nanowires containing angstrom scale twins. Nat. Commun. 4, 1-8 (2013).

9. Lu, L., Shen, Y. F., Chen, X. H., Qian, L. H. \& Lu, K. Ultrahigh strength and high electrical conductivity in copper. Science 304, 422-426 (2004).

10. Lu, K., Lu, L. \& Suresh, S. Strengthening materials by engineering coherent internal boundaries at the nanoscale. Science 324, 349-352 (2009).

11. Li, X. Y., Wei, Y. J., Lu, L., Lu, K. \& Gao, H. J. Dislocation nucleation governed softening and maximum strength in nano-twinned metals. Nature 464, $877-880$ (2010)

12. Lu, L., Chen, X., Huang, X. \& Lu, K. Revealing the maximum strength in nanotwinned copper. Science 323, 607-610 (2009).

13. Christian, J. W. \& Mahajan, S. Deformation twinning. Prog. Mater. Sci. 39, $1-157$ (1995).

14. Jin, Z. H. et al. The interaction mechanism of screw dislocations with coherent twin boundaries in different face-centred cubic metals. Scripta Mater. 54, 1163-1168 (2006)

15. Pestman, B. J. \& de Hosson, J. T. M. Interactions between lattice dislocations and grain boundaries in $\mathrm{Ni3Al}$ investigated by means of in situ TEM and computer modelling experiments. Acta Metall. Mater. 40, 2511-2521 (1992).

16. Meyers, M. A., Mishra, A. \& Benson, D. J. Mechanical properties of nanocrystalline materials. Prog. Mater. Sci. 51, 427-556 (2006).

17. You, Z. S. et al. Plastic anisotropy and associated deformation mechanisms in nanotwinned metals. Acta Mater. 61, 217-227 (2013).

18. Jang, D. C., Li, X. Y., Gao, H. J. \& Greer, J. R. Deformation mechanisms in nanotwinned metal nanopillars. Nat. Nanotech. 7, 594-601 (2012).

19. Zhang, Y. W. Nanotwins only. Nat. Nanotech. 7, 551-552 (2012).

20. Zhang, P., Duan, Q. Q., Li, S. X. \& Zhang, Z. F. Cyclic deformation and fatigue cracking behavior of polycrystalline $\mathrm{Cu}, \mathrm{Cu}-10 \mathrm{wt} \% \mathrm{Zn}$ and $\mathrm{Cu}-32 \mathrm{wt} \% \mathrm{Zn}$. Philos. Mag. 88, 2487-2503 (2008).

21. Zhang, Z. J., Li, L. L., Zhang, P. \& Zhang, Z. F. Fatigue cracking at twin boundary: effect of dislocation reactions. Appl. Phys. Lett. 101, 011907 (2012).

22. Zhang, Z. J., Zhang, P., Li, L. L. \& Zhang, Z. F. Fatigue cracking at twin boundaries: effects of crystallographic orientation and stacking fault energy. Acta Mater. 60, 3113-3127 (2012).

23. Li, L. L. et al. Microcompression and cyclic deformation behaviors of coaxia copper bicrystals with a single twin boundary. Scripta Mater. 69, 199-202 (2013)

24. Li, L. L., Zhang, P., Zhang, Z. J. \& Zhang, Z. F. Effect of crystallographic orientation and grain boundary character on fatigue cracking behaviors of coaxial copper bicrystals. Acta Mater. 61, 425-438 (2013).

25. Laufer, E. E. \& Roberts, W. N. Dislocations and persistent slip bands in fatigued copper. Philos. Mag. 14, 65-78 (1966).

26. Saletore, M. \& Taggart, R. Role of deformation bands in fatigue crack nucleation and propagation in copper crystals. Mater. Sci. Eng. 36, 259-270 (1978).

27. Zhang, Z. F., Wang, Z. G. \& Sun, Z. M. Evolution and microstructural characteristics of deformation bands in fatigued copper single crystals. Acta Mater. 49, 2875-2886 (2001).

28. Li, P., Li, S. X., Wang, Z. G. \& Zhang, Z. F. Formation mechanisms of cyclic saturation dislocation patterns in [0 $\left.0 \begin{array}{ll}0 & 1\end{array}\right],\left[\begin{array}{lll}0 & 1 & 1\end{array}\right]$ and [ $\left[\begin{array}{lll}-1 & 1 & 1\end{array}\right]$ copper single crystals. Acta Mater. 58, 3281-3294 (2010).

29. Heinz, A. \& Neumann, P. Crack initiation during high cycle fatigue of an austenitic steel. Acta Metall. Mater. 38, 1933-1940 (1990).

30. Ma, J. AFM study of the morphology and micro-strain of slip bands in twin under cyclic deformation. Mater. Sci. Eng. A457, 63-68 (2007).

31. Lepistö, T. K. \& Kettunen, P. O. Comparison of the cyclic stress-strain behaviour of single- and $\langle 111\rangle$ multiple-slip-orientated copper single crystal. Mater. Sci. Eng. 83, 1-15 (1986).
32. Li, P., Li, S. X., Wang, Z. G. \& Zhang, Z. F. Fundamental factors on formation mechanisms of dislocation arrangement in cyclically deformed fcc single crystals. Prog. Mater. Sci. 56, 328-377 (2011).

33. Wang, Z. R. \& Margolin, H. Mechanism for the formation of high cycle fatigue cracks at fcc annealing twin boundaries. Metall. Trans. 16A, 873-880 (1985).

34. Zhu, Y. T. et al. Dislocation-twin interactions in nanocrystalline fcc metals. Acta Mater. 59, 812-821 (2011).

35. Zhai, T. G., Lin, S. \& Xiao, J. M. Influence of non-geometric effect of PSB on crack initiation in aluminium single crystal. Acta Metall. Mater. 38, 1687-1692 (1990).

36. Meyers, M. A. \& Chawla, K. K. Mechanical Behavior of Materials (Prentice Hall: Upper Saddle River, NJ, 1999).

37. Ma, E. et al. Strain hardening and large tensile elongation in ultrahigh-strength nanotwinned copper. Appl. Phys. Lett. 85, 4932 (2004).

38. Zhang, X. et al. High-strength sputter-deposited Cu foils with preferred orientation of nanoscale growth twins. Appl. Phys. Lett. 88, 173116 (2006).

39. Blochwitz, C. \& Tirschler, W. Influence of texture on twin boundary cracks in fatigued austenitic stainless steel. Mater. Sci. Eng. A 339, 318-327 (2003).

40. Yan, B. D., Hunsche, A., Nuumann, P. \& Laird, C. Loop patch behavior as affected by incremental loading and cyclic frequency in fatigue. Mater. Sci. Eng. 79, 9-14 (1986).

41. Melisova, D., Weiss, B. \& Stickler, R. Nucleation of persistent slip bands in $\mathrm{Cu}$ single crystals under stress controlled cycling. Scripta Mater. 36, 1061-1066 (1997).

42. Gutierrez-Urrutia, I., Zaefferer, S. \& Raabe, D. Electron channeling contrast imaging of twins and dislocations in twinning-induced plasticity steels under controlled diffraction conditions in a scanning electron microscope. Scripta Mater. 61, 737-740 (2009).

43. Ahmed, J., Wilkinson, A. J. \& Roberts, S. G. Characterizing dislocation structures in bulk fatigued copper single crystals using electron channelling contrast imaging (ECCI). Philos. Mag. Lett. 76, 237-245 (1997).

44. Dudarev, S. L., Ahmed, J., Hirsch, P. B. \& Wilkinson, A. J. Decoherence in electron backscattering by kinked dislocations. Acta Crystallogr. A 55, 234-245 (1999).

45. Zhang, Z. F. \& Wang, Z. G. Investigations of dislocation patterns within grains and near grain boundaries in copper by the electron channelling contrast technique in scanning electron microscopy. Philos. Mag. Lett. 78, 105-113 (1998).

46. Zauter, R. et al. Electron channelling contrast as a supplementary method for microstructural investigations in deformed metals. Philos. Mag. A 66, 425-436 (1992).

\section{Acknowledgements}

This work was financially supported by the National Natural Science Foundation of China (NSFC) under grant Nos 50890173, 51171194 and the National Basic Research Program of China under grant No. 2010CB631006.

\section{Authors contributions}

Z.F.Z. conceived and designed the research plan. L.L.L. carried out the cyclicdeformation experiments of the bulk bicrystals. L.L.L., Z.J.Z., P.Z. and Z.F.Z. analysed the data and wrote the paper. Z.G.W. and Z.F.Z. revised the paper. All authors contributed to the scientific discussions.

\section{Additional information}

Supplementary Information accompanies this paper at http://www.nature.com/ naturecommunications

Competing financial interests: The authors declare no competing financial interests

Reprints and permission information is available online at http://npg.nature.com/ reprintsandpermissions/

How to cite this article: Li, L. L. et al. Controllable fatigue cracking mechanisms of copper bicrystals with a coherent twin boundary. Nat. Commun. 5:3536 doi: $10.1038 /$ ncomms4536 (2014). 